PROCEEDINGS OF THE AMERICAN MATHEMATICAL SOCIETY

Volume 124, Number 1, January 1996

\title{
ON THE UNIQUE RANGE SET OF MEROMORPHIC FUNCTIONS
}

\author{
PING LI AND CHUNG-CHUN YANG
}

(Communicated by Albert Baernstein II)

\begin{abstract}
This paper studies the unique range set of meromorphic functions and shows that there exists a finite set $S$ such that for any two nonconstant meromorphic functions $f$ and $g$ the condition $E_{f}(S)=E_{g}(S)$ implies $f \equiv g$. As a special case this also answers an open question posed by Gross (1977) about entire functions and improves some results obtained recently by Yi.
\end{abstract}

\section{INTRODUCTION}

Let $f$ be a nonconstant meromorphic function on the complex plane $C$ and $S$ be a subset of distinct elements in $C$. Define

$$
E_{f}(S)=\bigcup_{a \in S}\{z \mid f(z)-a=0\},
$$

here a zero of $f(z)-a$ of multiplicity $m$ appears $m$ times in $E_{f}(S)$. In 1976 Gross proved [1] that there exist three finite sets $S_{j}(j=1,2,3)$ such that for any two nonconstant entire functions $f$ and $g$ if $E_{f}\left(S_{j}\right)=E_{g}\left(S_{j}\right)(j=1,2,3)$, then $f \equiv g$. In the same paper Gross posed the following problem: Can one find two (or possibly even one) finite sets $S_{j}(j=1,2)$ such that any two entire functions $f$ and $g$ satisfying $E_{f}\left(S_{j}\right)=E_{g}\left(S_{j}\right)(j=1,2)$ must be identical? In 1982, F. Gross and C. C. Yang proved the following result.

Theorem A ([2]). Let $T=\left\{z \mid e^{z}+z=0\right\}$. Let $f$ and $g$ be two nonconstant entire functions. If $E_{f}(T)=E_{g}(T)$, then $f \equiv g$.

In [2] the set $S$ such that for any two nonconstant entire functions $f$ and $g$ the condition $E_{f}(S)=E_{g}(S)$ implies $f \equiv g$ is called a unique range set (URS, in brief) of entire functions. A similar definition for meromorphic functions can be defined. Note that the set $T=\left\{z \mid e^{z}+z=0\right\}$ contains an infinite number of elements. Recently, Yi [6] exhibited a finite unique range set of entire functions which gave a positive answer to Gross's problem. He proved

Theorem B. Let $n \geq 15, n>m \geq 5$ with $n$ and $m$ having no common factors. Let $a$ and $b$ be two nonzero constants such that the algebraic equation $z^{n}+a z^{m}+b=0$ has no multiple roots. Then the set $S=\left\{z \mid z^{n}+a z^{m}+b=0\right\}$ is a URS of entire functions.

Received by the editors March 21, 1994 and, in revised form, July 26, 1994.

1991 Mathematics Subject Classification. Primary 30D35.

Key words and phrases. Meromorphic function, entire function, unique range set.

(C)1996 American Mathematical Society 
In this paper, we shall exhibit, among other results, a finite URS of meromorphic functions with 19 elements and a URS of entire functions with nine elements.

Theorem 1. Let $m \geq 2, n>4 m+10$ with $n$ and $n-m$ having no common factors. Let $a$ and $b$ be two nonzero constants such that the algebraic equation $z^{n}+a z^{n-m}+b=0$ has no multiple roots. Let $S=\left\{z \mid z^{n}+a z^{n-m}+b=0\right\}$. Then for any two nonconstant meromorphic functions $f$ and $g$, the condition $E_{f}(S)=E_{g}(S)$ implies $f \equiv g$.

Theorem 2. Let $m \geq 2, n>4 m+6$ with $n$ and $n-m$ having no common factors. Let $a, b$ and $S$ be as in Theorem 1. Then for any two nonconstant meromorphic functions $f$ and $g$, the conditions $E_{f}(S)=E_{g}(S)$ and $E_{f}\{\infty\}=E_{g}\{\infty\}$ imply $f \equiv g$.

Theorem 3. Let $m \geq 1, n>4 m+4$ with $n$ and $n-m$ having no common factors. Let $a, b$ and $S$ be as in Theorem 1. Then for any two nonconstant entire functions $f$ and $g$, the condition $E_{f}(S)=E_{g}(S)$ implies $f \equiv g$.

The main tool will be Nevanlinna's theory of meromorphic functions, and it is assumed that the reader is familiar with its basic notation and results (see Hayman [4]). In the sequel the letter $E$ will be used to denote a set of $r$ values of finite linear measure.

\section{Some Lemmas}

The following lemmas will be needed in the proof of our theorems.

Lemma 1 ([7]). Let $f$ and $g$ be two nonconstant meromorphic functions, and $c_{1}, c_{2}$, and $c_{3}$ be nonzero constants. If $c_{1} f+c_{2} g \equiv c_{3}$, then

$$
T(r, f)<\bar{N}\left(r, \frac{1}{f}\right)+\bar{N}\left(r, \frac{1}{g}\right)+\bar{N}(r, f)+S(r, f) .
$$

Here and in the sequel $S(r, f)$ denotes the quantity $o(T(r, f)), r \rightarrow \infty, r \notin E$.

Lemma 2. Let $f_{1}, f_{2}$, and $f_{3}$ be nonconstant meromorphic functions and $f_{1}+f_{2}+$ $f_{3} \equiv 1$. If $f_{1}, f_{2}, f_{3}$ are linearly independent, then

$$
T\left(r, f_{1}\right)<2 \sum_{i=1}^{3} \bar{N}\left(r, \frac{1}{f_{i}}\right)+\sum_{i=1}^{3} \bar{N}\left(r, f_{i}\right)+o(T(r))
$$

where $T(r)=\max _{1 \leq i \leq 3}\left\{T\left(r, f_{i}\right)\right\}$ and $r \notin E$.

Proof. By the proof of a generalization of Borel's theorem (a generalization of Picard's theorem) by Nevanlinna [3] (page 70), we have

$$
T\left(r, f_{1}\right)<\sum_{i=1}^{3} N\left(r, \frac{1}{f_{i}}\right)-\sum_{i=2}^{3} N\left(r, f_{i}\right)+N(r, D)-N\left(r, \frac{1}{D}\right)+o(T(r)),
$$

where $D$ is the Wronskian of $f_{1}, f_{2}$, and $f_{3}$, i.e.,

$$
D=\left|\begin{array}{ccc}
f_{1} & f_{2} & f_{3} \\
f_{1}^{\prime} & f_{2}^{\prime} & f_{3}^{\prime} \\
f_{1}^{\prime \prime} & f_{2}^{\prime \prime} & f_{3}^{\prime \prime}
\end{array}\right|
$$

Since $f_{1}+f_{2}+f_{3} \equiv 1$, we have

$$
D=f_{2}^{\prime} f_{3}^{\prime \prime}-f_{2}^{\prime \prime} f_{3}^{\prime}=-\left(f_{1}^{\prime} f_{3}^{\prime \prime}-f_{1}^{\prime \prime} f_{3}^{\prime}\right)=f_{1}^{\prime} f_{2}^{\prime \prime}-f_{1}^{\prime \prime} f_{2}^{\prime} \text {. }
$$


Write

$$
N(r)=\sum_{i=1}^{3} N\left(r, \frac{1}{f_{i}}\right)-\sum_{i=2}^{3} N\left(r, f_{i}\right)+N(r, D)-N\left(r, \frac{1}{D}\right)
$$

and

$$
N^{*}(r)=2 \sum_{i=1}^{3} \bar{N}\left(r, \frac{1}{f_{i}}\right)+\sum_{i=1}^{3} \bar{N}\left(r, f_{i}\right) .
$$

Thus clearly Lemma 2 follows immediately from the inequality

$$
N(r) \leq N^{*}(r),
$$

which is to be shown next.

For a given meromorphic function $f$ and a complex number $a \in \bar{C}$, we define

$$
\mu_{f}^{a}(z)= \begin{cases}m, & z \text { is an } a \text {-point of } f \text { with multiplicity } m \geq 1 \\ 0, & z \text { is not an } a \text {-point of } f\end{cases}
$$

and

$$
\bar{\mu}_{f}^{a}(z)= \begin{cases}1, & z \text { is an } a \text {-point of } f \text { with multiplicity } m \geq 1 \\ 0, & z \text { is not an } a \text {-point of } f\end{cases}
$$

Let

$$
\mu=\mu_{f_{1}}^{0}+\mu_{f_{2}}^{0}+\mu_{f_{3}}^{0}-\mu_{f_{2}}^{\infty}-\mu_{f_{3}}^{\infty}+\mu_{D}^{\infty}-\mu_{D}^{0}
$$

and

$$
\mu^{*}=2 \bar{\mu}_{f_{1}}^{0}+2 \bar{\mu}_{f_{2}}^{0}+2 \bar{\mu}_{f_{3}}^{0}+\bar{\mu}_{f_{1}}^{\infty}+\bar{\mu}_{f_{2}}^{\infty}+\bar{\mu}_{f_{3}}^{\infty} .
$$

Thus inequality (2) follows from $\mu(z) \leq \mu^{*}(z)$ for any $z$. To prove this, we consider the following five cases for an arbitrary point $z \in C$.

Case 1. $z$ is a zero-point of $f_{i}(i=1,2,3)$ with multiplicity $m_{i} \geq 0$.

Case 2. $z$ is a zero-point of $f_{1}$ with multiplicity $m \geq 1$ and a pole of $f_{2}$ and $f_{3}$ with multiplicity $k \geq 1$.

Case 3. $z$ is a zero-point of $f_{2}$ with multiplicity $m \geq 1$ and a pole of $f_{1}$ and $f_{3}$ with multiplicity $k \geq 1$.

Case 4. $z$ is a zero-point of $f_{3}$ with multiplicity $m \geq 1$ and a pole of $f_{1}$ and $f_{2}$ with multiplicity $k \geq 1$.

Case 5. $z$ is a pole of $D$ but not a zero of $f_{1}, f_{2}$, and $f_{3}$.

In each case we can verify that the inequality $\mu(z) \leq \mu^{*}(z)$ holds. For instance, take Case 2; then we have $\mu_{f_{1}}^{0}(z)=m, \mu_{f_{2}}^{0}=\mu_{f_{3}}^{0}(z)=0, \mu_{f_{2}}^{\infty}(z)=\mu_{f_{3}}^{\infty}(z)=k$. Thus $\mu^{*}(z)=4$.

If $k-m+3>0$, then from (1), $z$ is a pole of $D$ with multiplicity at most $k-m+3$. This means that $\mu_{D}^{\infty} \leq k-m+3$. It follows that

$$
\mu(z) \leq m-2 k+(k-m+3)=3-k \leq 2<\mu^{*}(z) .
$$

If $k-m+3 \leq 0$, then from (1) $z$ is a zero of $D$ with multiplicity at least $m-k-3$. This means that $\mu_{D}^{\infty}(z)=0$ and $\mu_{D}^{0}(z) \geq m-k-3$. Hence

$$
\mu(z) \leq m-2 k-(m-k-3)=3-k \leq 2<\mu^{*}(z) .
$$

The remaining cases can be proved in a similar manner. This also completes the proof of the lemma. 
Lemma 3 ([5]). Let $f$ be a meromorphic function, and

$$
P(f)=a_{0} f^{n}+a_{1} f^{n-1}+\cdots+a_{n}
$$

be a polynomial in $f$ of degree $n$, where $a_{0}(\neq 0), a_{1}, \ldots, a_{n}$ are finite complex numbers. Then

$$
T(r, P(f))=n T(r, f)+S(r, f) .
$$

\section{Proof of Theorem 1}

Let $r_{1}, r_{2}, \ldots, r_{n}$ be the roots of equation $z^{n}+a z^{n-m}+b=0$. Since $E_{f}(S)=$ $E_{g}(S)$, we have from Nevanlinna's second fundamental theorem

$$
\begin{aligned}
(n-2) T(r, g) & <\sum_{k=1}^{n} \bar{N}\left(r, \frac{1}{g-r_{k}}\right)+S(r, g) \\
& =\sum_{k=1}^{n} \bar{N}\left(r, \frac{1}{f-r_{k}}\right)+S(r, g) \\
& \leq n T(r, f)+S(r, g) .
\end{aligned}
$$

It follows that

$$
T(r, g) \leq \frac{n}{n-2} T(r, f)+S(r, g) .
$$

Similarly the following inequality holds:

$$
T(r, f) \leq \frac{n}{n-2} T(r, g)+S(r, f) .
$$

In the sequel we use $S(r)$ to express either $S(r, f)$ or $S(r, g)$.

Consider now the following meromorphic function

$$
\psi=\frac{f^{n}+a f^{n-m}+b}{g^{n}+a g^{n-m}+b} .
$$

The condition $E_{f}(S)=E_{g}(S)$ ensures that the zeros of $\psi$ come from the poles of $g$, and the poles of $\psi$ come from the poles of $f$. This means that the following inequalities hold:

$$
\bar{N}\left(r, \frac{1}{\psi}\right) \leq \bar{N}(r, g)
$$

and

$$
\bar{N}(r, \psi) \leq \bar{N}(r, f)
$$

Let

$$
f_{1}=-\frac{1}{b} f^{n-m}\left(f^{m}+a\right), \quad f_{2}=\frac{1}{b} \psi g^{n-m}\left(g^{m}+a\right), \quad f_{3}=\psi .
$$

Then $f_{1}, f_{2}$, and $f_{3}$ are meromorphic functions and $f_{1}$ is not a constant. From (3), we have

$$
f_{1}+f_{2}+f_{3} \equiv 1
$$

Now we distinguish two cases. 
Case 1: $f_{3}$ is not a constant. If $f_{1}$ and $f_{2}$ are linearly dependent, then $f_{2}=c f_{1}, c \neq$ -1 . From (9) we have

$$
(1+c) f_{1}+f_{3} \equiv 1
$$

By using Lemma 1 and Lemma 3 together with the inequalities (3) and (6), we deduce

$$
\begin{aligned}
n T(r, f) & =T\left(r, f_{1}\right)+S(r) \\
& <\bar{N}\left(r, \frac{1}{f_{1}}\right)+\bar{N}\left(r, \frac{1}{f_{3}}\right)+\bar{N}\left(r, f_{1}\right)+S(r) \\
& \leq \bar{N}\left(r, \frac{1}{f}\right)+\bar{N}\left(r, \frac{1}{f^{m}+a}\right)+\bar{N}(r, g)+\bar{N}(r, f)+S(r) \\
& \leq(m+2) T(r, f)+T(r, g)+S(r) \\
& \leq\left(m+2+\frac{n}{n-2}\right) T(r, f)+S(r) \\
& =\left(m+3+\frac{2}{n-2}\right) T(r, f)+S(r),
\end{aligned}
$$

which is contradictory to $n>4 m+10$. Hence $f_{1}$ and $f_{2}$ must be linearly independent.

If $f_{1}, f_{2}$, and $f_{3}$ are linearly independent and $f_{2}$ is not a constant, then by using Lemma 2 we have

$$
\begin{aligned}
T\left(r, f_{1}\right)< & 2 \bar{N}\left(r, \frac{1}{f_{1}}\right)+2 \bar{N}\left(r, \frac{1}{f_{2}}\right)+2 \bar{N}\left(r, \frac{1}{f_{3}}\right) \\
& +\bar{N}\left(r, f_{1}\right)+\bar{N}\left(r, f_{2}\right)+\bar{N}\left(r, f_{3}\right)+S(r) .
\end{aligned}
$$

From the identities (5) and (8), we can easily see that the zeros of $f_{2}$ cannot come from the zeros of $\psi$, and the poles of $f_{2}$ must come from the poles of $f$. By the above inequality and Lemma 3 together with (6), (7) and (8), we deduce that

$$
\begin{aligned}
n T(r, f)< & 2 \bar{N}\left(r, \frac{1}{f}\right)+2 \bar{N}\left(r, \frac{1}{f^{m}+a}\right)+2 \bar{N}\left(r, \frac{1}{g}\right)+2 \bar{N}\left(r, \frac{1}{g^{m}+a}\right) \\
& +2 \bar{N}(r, g)+\bar{N}(r, f)+\bar{N}(r, f)+\bar{N}(r, f)+S(r) \\
\leq & (2 m+5) T(r, f)+2(m+2) T(r, g)+S(r) \\
\leq & {\left[(2 m+5)+2(m+2) \frac{n}{n-2}\right] T(r, f)+S(r) } \\
= & \left(4 m+9+\frac{4 m+8}{n-2}\right) T(r, f)+S(r) .
\end{aligned}
$$

This contradicts the assumption $n>4 m+10$. It follows that when $f_{1}, f_{2}$, and $f_{3}$ are linearly independent, $f_{2}$ must be constant and $f_{2} \neq-1$, i.e. $f_{1}+f_{3}=1-f_{2}$ is a nonzero constant. By Lemma 1,

$$
T\left(r, f_{1}\right)<\bar{N}\left(r, \frac{1}{f_{1}}\right)+\bar{N}\left(r, \frac{1}{f_{3}}\right)+\bar{N}\left(r, f_{1}\right)+S(r) .
$$

This leads to

$$
n T(r, f) \leq\left(m+3+\frac{2}{n-2}\right) T(r, f)+S(r)
$$


which is a contradiction to $n>4 m+10$.

If $f_{1}, f_{2}$, and $f_{3}$ are linearly dependent, then there exist three constants $c_{1}, c_{2}$, and $c_{3}$, at least one of them is not zero, such that

$$
c_{1} f_{1}+c_{2} f_{2}+c_{3} f_{3} \equiv 0 .
$$

This and the fact that $f_{1}, f_{2}$ are linearly independent imply $c_{3} \neq 0$. So

$$
c_{1} \frac{f_{1}}{\psi}+c_{2} \frac{f_{2}}{\psi}=-c_{3} .
$$

If $c_{1}=0$, then $g^{n-m}\left(g^{m}+a\right)$; hence $g$ is a constant. This is impossible.

If $c_{2}=0$, then

$$
\frac{c_{1}}{b} f^{n-m}\left(f^{m}+a\right)=c_{3} \psi .
$$

Let $s_{0}=0, s_{1}, \ldots, s_{m}$ be the distinct roots of the equation $z^{n}+a z^{n-m}=0$. Then (12) shows that any $s_{j}$-point of $f$ must be a zero of $\psi$ and hence a pole of $g$. But from (5) and (12) one can see that the multiplicity of any zero of $\psi$ is at least $n$, so the multiplicity of an $s_{j}$-point $(j \neq 0)$ of $f$ is at least $n$ and at least $m$ for an $s_{0}$-point of $f$. Hence, we have

$$
\Theta\left(s_{j}, f\right)=1-\varlimsup_{r \rightarrow \infty} \frac{\bar{N}\left(r, \frac{1}{f-s_{j}}\right)}{T(r, f)} \geq 1-\varlimsup_{r \rightarrow \infty} \frac{\bar{N}\left(r, \frac{1}{f-s_{j}}\right)}{N\left(r, \frac{1}{f-s_{j}}\right)} \geq 1-\frac{1}{n},
$$

$j=1,2, \ldots, m$, and

$$
\Theta\left(s_{0}, f\right) \geq 1-\frac{1}{m}
$$

Again by the second fundamental theorem about the deficiencies of meromorphic functions, we have

$$
1-\frac{1}{m}+m\left(1-\frac{1}{n}\right) \leq \sum_{j=0}^{m} \Theta\left(s_{j}, f\right) \leq 2 .
$$

This is impossible because $m \geq 2, n>4 m+10$.

Now that we have obtained $c_{1} \neq 0, c_{2} \neq 0, c_{3} \neq 0$, by Lemma 1 and (11)

$$
\begin{aligned}
T\left(r, \frac{f_{2}}{\psi}\right) & <\bar{N}\left(r, \frac{\psi}{f_{2}}\right)+\bar{N}\left(r, \frac{\psi}{f_{1}}\right)+\bar{N}\left(r, \frac{f_{2}}{\psi}\right)+S(r) \\
& <\bar{N}\left(r, \frac{\psi}{f_{2}}\right)+\bar{N}(r, \psi)+\bar{N}\left(r, \frac{1}{f_{1}}\right)+\bar{N}\left(r, \frac{f_{2}}{\psi}\right)+S(r) .
\end{aligned}
$$

Hence from Lemma 3 and (7) and (8), we have

$$
\begin{aligned}
n T(r, g)< & \bar{N}\left(r, \frac{1}{g}\right)+\bar{N}\left(r, \frac{1}{g^{m}+a}\right)+\bar{N}(r, f) \\
& +\bar{N}\left(r, \frac{1}{f^{m}+a}\right)+\bar{N}(r, g)+S(r) \\
\leq & (m+2) T(r, g)+(m+2) T(r, f)+S(r) \\
\leq & (m+2)\left(1+\frac{n}{n-2}\right) T(r, f)+S(r),
\end{aligned}
$$

which is a contradiction to $n>4 m+10$.

We can rule out Case 1. 
Case 2: $f_{3}$ is a constant. In this case, $f_{2}$ cannot be a constant. From (5), we have

$$
T(r, f)=T(r, g)+S(r) .
$$

If $f_{3} \neq 1$, then $f_{1}+f_{2}=1-f_{3} \neq 0$. By Lemma 1

$$
T\left(r, f_{1}\right)<\bar{N}\left(r, \frac{1}{f_{1}}\right)+\bar{N}\left(r, \frac{1}{f_{2}}\right)+\bar{N}\left(r, f_{1}\right)+S(r) .
$$

That is,

$$
\begin{aligned}
n T(r, f)< & \bar{N}\left(r, \frac{1}{f_{1}}\right)+\bar{N}\left(r, \frac{1}{f^{m}+a}\right)+\bar{N}\left(r, \frac{1}{g}\right) \\
& +\bar{N}\left(r, \frac{1}{g^{m}+a}\right)+\bar{N}(r, f)+S(r) \\
< & (m+2) T(r, f)+(m+1) T(r, g)+S(r) \\
= & (2 m+3) T(r, f)+S(r) .
\end{aligned}
$$

This contradicts the assumption that $n>4 m+10$.

If $f_{3}=1$, then from (5) we get

$$
g^{m}\left(h^{n}-1\right)=-a\left(h^{n-m}-1\right)
$$

where $h=f / g$ is a meromorphic function. Further (14) can be rewritten as

$$
g^{m}\left(h-u_{1}\right)\left(h-u_{2}\right) \cdots\left(h-u_{n}\right)=-a\left(h^{n-m}-1\right)
$$

where $u_{j}=e^{i 2 j \pi / n}, j=1,2, \ldots, n$. Since $n$ and $n-m$ have no common factors, we see that $u_{j}^{n-m}-1 \neq 0, j=1, \ldots, n-1$. Hence from (15) the multiplicity of a $u_{j}$-point of $h$ is at least $m$. Suppose that $h$ is not a constant, then we have

$$
\begin{aligned}
\Theta\left(u_{j}, h\right) & =1-\varlimsup_{r \rightarrow \infty} \frac{\bar{N}\left(r, \frac{1}{h-u_{j}}\right)}{T(r, h)} \\
& \geq 1-\varlimsup_{r \rightarrow \infty} \frac{\bar{N}\left(r, \frac{1}{h-u_{j}}\right)}{N\left(r, \frac{1}{h-u_{j}}\right)} \geq 1-\frac{1}{m}, \quad j=1, \ldots, n-1 .
\end{aligned}
$$

Thus

$$
(n-1)\left(1-\frac{1}{m}\right) \leq \sum_{j=1}^{n-1} \Theta\left(u_{j}, h\right) \leq 2,
$$

which contradicts $m \geq 2$ and $n>4 m+10$. This shows that $h$ must be a constant. Furthermore from (14) we can see that $h$ must be equal to 1 . Otherwise we will deduce that $g$ is a constant. Hence $f \equiv g$. This completes the proof of Theorem 1 .

Note that the function $\psi$ in (5) will assume the form $e^{\alpha}$ with $\alpha$ being an entire function under the assumptions of Theorem 2 and Theorem 3. Furthermore under the assumption of Theorem 3 the inequalities (3) and (4) will be replaced by

$$
T(r, g) \leq \frac{n}{n-1} T(r, f)+S(r, g)
$$

and

$$
T(r, f) \leq \frac{n}{n-1} T(r, g)+S(r, f)
$$


respectively, and we can then prove these two theorems immediately following the same procedure of the proof of Theorem 1 .

Example 1. The set $S=\left\{z \mid z^{19}-z^{17}+1=0\right\}$ is a URS of meromorphic functions with 19 elements.

Example 2. The set $S=\left\{z \mid z^{9}-z^{8}+1=0\right\}$ is a URS of entire functions with nine elements.

\section{Concluding Remarks}

We would like to pose the following problems about the unique range set of meromorphic functions and entire functions for further investigations.

Problem 1. Can one find a URS of entire functions with fewer than nine elements? What is the smallest cardinality for a URS of entire functions?

Problem 2. Can one find a URS of meromorphic functions with fewer than 19 elements? What is the smallest cardinality for a URS of meromorphic functions?

Now we introduce the following notation:

$$
\begin{aligned}
U_{M} & =\{S \mid S \text { is a URS of meromorphic functions }\}, \\
U_{E} & =\{S \mid S \text { is a URS of entire functions }\}, \\
\lambda_{M} & =\min \left\{n(S) \mid S \in U_{M}\right\}, \\
\lambda_{E} & =\min \left\{n(S) \mid S \in U_{E}\right\},
\end{aligned}
$$

where $n(S)$ denotes the cardinal number of the set $S$. Obviously,

$$
\lambda_{E} \leq \lambda_{M}
$$

Example 1 and Example 2 show that $\lambda_{E} \leq 9$ and $\lambda_{M} \leq 19$, respectively. We claim

Theorem 4. $\lambda_{E} \geq 4$.

Proof. Let us consider the two entire functions

$$
f=\frac{\omega_{2} e^{h}}{\omega_{2}-\omega_{1}}+\frac{t \omega_{1} e^{-h}}{\omega_{2}-\omega_{1}}+\frac{1}{3}\left(a_{1}+a_{2}+a_{3}\right)
$$

and

$$
g=\frac{e^{h}}{\omega_{2}-\omega_{1}}+\frac{t e^{-h}}{\omega_{2}-\omega_{1}}+\frac{1}{3}\left(a_{1}+a_{2}+a_{3}\right)
$$

where $h$ is any nonconstant entire function, $a_{1}, a_{2}$, and $a_{3}$ are three finite distinct complex numbers, and

$$
\begin{gathered}
t=a_{1} a_{2}+a_{1} a_{2}+a_{2} a_{3}-\frac{1}{3}\left(a_{1}+a_{2}+a_{3}\right)^{2}, \\
\omega_{1}=e^{i 2 \pi / 3}, \quad \omega_{2}=e^{i 4 \pi / 3} .
\end{gathered}
$$

It is easy to verify that

$$
\left(f-a_{1}\right)\left(f-a_{2}\right)\left(f-a_{3}\right) \equiv\left(g-a_{1}\right)\left(g-a_{2}\right)\left(g-a_{3}\right),
$$

which shows $E_{f}\left\{a_{1}, a_{2}, a_{3}\right\}=E_{g}\left\{a_{1}, a_{2}, a_{3}\right\}$, but obviously $f$ is not identically equal to $g$. Hence $\lambda_{E} \geq 4$.

We conjecture that $\lambda_{E}=4$ is the answer to Problem 1 . 


\section{REFERENCES}

1. F. Gross, Complex analysis, Lecture Notes in Math., vol. 599, Springer, Berlin and New York, 1977 pp. 51-69

2. F. Gross and C. C. Yang, On preimage and range sets of meromorphic functions, Proc. Japan Acad. Ser. A Math. Sci. 58 (1982), 17-20 MR 83d:30027

3. F. Gross, Factorizations of meromorphic functions, U.S. Government Printing Office Publication, Washington D.C., 1972.

4. W. K. Hayman, Meromorphic functions, Clarendon Press, Oxford, 1964. MR 29:6019

5. C. C. Yang, On deficiencies of differential polynomials, II Math. Z. 125 (1972) 107-112. MR 45:3710

6. Hongxun Yi, On a problem of Gross, Sci. China Ser. A 11 (24) (1994), 1137-1144.

7. $ـ$ Meromorphic functions that share three values, Chinese Ann. Math. Ser. A 9 (1988) 434-439. MR 90f:30039

Department of Mathematics, Hong Kong University of Science and Technology, Clear Water Bay, Kowloon, Hong Kong

E-mail address: mayang@usthk.bitnet 